\title{
Dysregulation of MALAT1 and miR-619-5p as a prognostic indicator in advanced colorectal carcinoma
}

\author{
GU QIU ${ }^{1}$, XIU-BING ZHANG ${ }^{2}$, SHU-QING ZHANG ${ }^{1}$, PEI-LONG LIU ${ }^{1}$, \\ WEI WU ${ }^{3}$, JIN-YE ZHANG ${ }^{4}$ and SHI-RONG DAI ${ }^{1}$
}

\begin{abstract}
Departments of ${ }^{1}$ Clinical Laboratories and ${ }^{2}$ Internal Medicine-Oncology, Nantong Second People's Hospital, Nantong, Jiangsu 226002; ${ }^{3}$ Department of Experimental Center, Affiliated Hospital of Nantong University, Nantong, Jiangsu 226001;

${ }^{4}$ Department of Clinical Laboratories, Nantong Tumor Hospital, Nantong, Jiangsu 226018, P.R. China
\end{abstract}

Received January 25, 2015; Accepted March 24, 2016

DOI: $10.3892 / \mathrm{ol} .2016 .5312$

\begin{abstract}
The present study aimed to detect the expression of metastasis associated lung adenocarcinoma transcript 1 (MALAT1) and microRNA (miR)-619-5p in colorectal carcinoma (CRC), and to evaluate the significance of MALAT1 and miR-619-5p expression in the clinical diagnosis and prognosis of CRC. Quantitative polymerase chain reaction was used to detect MALAT1 and miR-619-5p expression in 120 colorectal carcinoma and 120 adjacent normal tissue samples. The expression levels of MALAT1 and miR-619-5p were significantly different between colorectal carcinoma and adjacent normal tissues $(\mathrm{P}<0.05)$. MALAT1 exhibited an average 2.52-fold increase in colorectal adenoma when compared with adjacent normal tissues, while miR-619-5p exhibited an average 5.79-fold decrease in colorectal adenoma when compared with adjacent normal tissues. There was a significant difference between the MALAT1 expression in CRC tissues obtained from men and women $(\mathrm{P}=0.027)$, and in tumor-node-metastasis (TNM) stage II and stage III lesions $(\mathrm{P}=0.019)$. MALAT1 expression was associated with lymphovascular invasion $(\mathrm{P}=0.047)$ and perineural invasion $(\mathrm{P}=0.012)$. In addition, miR-619-5p expression was also significantly different between men and women $(\mathrm{P}=0.032)$, and between TNM stage II and stage III lesions $(\mathrm{P}=0.012)$. miR-619-5p expression was also associated with lymphovascular invasion $(\mathrm{P}=0.023)$ and perineural invasion $(\mathrm{P}=0.009)$. Patients with high expression of MALAT1 and low expression of miR-619-5p demonstrated significantly shorter disease-free survival $(\mathrm{DFS})(\mathrm{P}=0.002)$ and overall survival $(\mathrm{OS})$ times $(\mathrm{P}=0.004)$ compared with patients with low MALAT1 expression and high miR-619-5p expression. Patients with perineural invasion demonstrated significantly
\end{abstract}

Correspondence to: Professor Shi-Rong Dai, Department of Clinical Laboratories, Nantong Second People's Hospital, Building 2, Xinglong Street 43, Nantong, Jiangsu 226002, P.R. China

E-mail: ntqg1103@sina.com

Key words: MALAT1, miR-619-5p, colorectal carcinoma, prognostic indicator shorter DFS $(\mathrm{P}=0.001)$ and OS times $(\mathrm{P}=0.003)$ compared with patients without perineural invasion. In addition, there was a negative correlation between MALAT1 expression and miR-619-5p expression ( $r=-0.415, \mathrm{P}=0.004)$ in CRC tissues. In conclusion, MALAT1 and miR-619-5p have potential for the molecular diagnosis of CRC patients, and combined assaying of MALAT1 and miR-619-5p may improve the accuracy of the diagnosis of CRC and act as a good prognostic indicator in CRC patients.

\section{Introduction}

Colorectal carcinoma (CRC) is a common clinical malignancy, with an incidence that ranks as the second most frequent malignant neoplasm in Western countries, and the incidence of colorectal cancer in China is also rising, becoming the most common malignancy (1). At present, the primary treatment for colorectal cancer is surgery, and early diagnosis and timely surgical treatment markedly improve the survival rate of patients. Therefore, identifying key factors involved in the development of cancer is necessary in order to not only aid the diagnosis of cancer, but also to act as a prognostic indicator in cancer patients.

Non-coding RNAs (ncRNAs) are found throughout the genome, although the function of ncRNAs is only partially understood. Numerous studies indicate that long ncRNAs (lncRNAs), which are $>200 \mathrm{nt}$ in length, and microRNAs (miRNAs or miRs), which are 22 nt in length, have various functions in the development and progression of cancer (2-8). Metastasis associated lung adenocarcinoma transcript 1 (MALAT1) is an abundant nucleus-restricted lncRNA, and previous studies have demonstrated that MALAT1 is upregulated in several solid tumors, and associated with cancer metastasis and recurrence (9-14). All these studies have revealed that MALAT1 plays an important role in promoting tumorigenesis, and tumors expressing a high level of MALAT1 appear to be more invasive and result in a poorer prognosis.

miRNAs have been reported to be involved in tumorigenesis $(15,16)$, acting as tumor suppressors, such as miRNA-15a, oncogenes, such as miRNA-21, or as promoters and suppressors of metastasis, such as miRNA-182 and miRNA-126, respectively. Other studies have described the altered 
expression of miRNAs in cancer tissues compared with normal tissues $(17,18)$, suggesting that these miRNAs may potentially indicate novel clinical diagnostic and prognostic markers. miRNA-619-5p (miR-619-5p) is a miRNA that binds with high affinity to the messenger (m)RNA of 1,215 genes, and miR-619-5p has binding sites in the coding sequences and untranslated regions (UTRs) of mRNAs (19).

Bioinformatics analysis indicated that miR-619-5p has several binding sites on the 3'-UTR of MALAT1. However, the association between MALAT1 and miR-619-5p in CRC is not well studied. In the present study, the correlations between the expression of MALAT1 and miR-619-5p was investigated, in addition to the association between the clinicopathological features and survival outcomes of patients with stage II and III CRC.

\section{Materials and methods}

Clinical samples. Paraffin-embedded tumors and adjacent normal tissue samples from 120 patients with CRC who underwent tumor resection at Nantong Second People's Hospital (Nantong, Jiangsu, China), Nantong Tumor Hospital (Nantong, Jiangsu, China) or Affiliated Hospital of Nantong University (Nantong, Jiangsu, China) between 2006 and 2010 were collected. All patients had not received chemotherapy or radiotherapy prior to surgery. Each tissue sample was obtained under sterile conditions by removing the CRC tissue and normal colon mucosa along the surgical margin. The present study was approved by the ethics committees of Nantong Second People's Hospital, Nantong Tumor Hospital, and Affiliated Hospital of Nantong University. All patients provided written informed consent.

Total RNA and miRNA isolation. Total RNA was extracted from tissues using TRIzol reagent (Takara Biotechnology Co., Ltd., Dalian, China) and complementary DNA was synthesized using PrimeScript ${ }^{\mathrm{TM}}$ RT Reagent kit with gDNA Eraser (Perfect Real Time) obtained from Takara Biotechnology Co., Ltd., according to the manufacturer's protocol. miRNA was extracted from the tissue using the Ambion mirVana ${ }^{\mathrm{TM}}$ miRNA Isolation kit (catalog no., AM1560; Thermo Fisher Scientific, Inc., Waltham, MA, USA). All preparation and handling steps for RNA isolation were performed under RNase-free conditions.

Quantitative polymerase chain reaction ( $q P C R$ ). All primers were designed and synthesized by Shanghai Sangon Biological Engineering Technology \& Services Co.,Ltd.(Shanghai,China), and the primer sequences were as follows: MALAT1 forward, 5'-AGGCGTTGTGCGTAGAGGA-3' and reverse, 5'-GGA TTTTTACCAACCACTCGC-3'; glyceraldehyde-3-phosphate dehydrogenase (GAPDH) forward, 5'-GGTGGTCTCCTC TGACTTCAACA-3' and reverse, 5'-CCAAATTCGTTGTCA TACCAGGAAATG-3'; miR-619-5p forward, 5'-GCUGGG AUUACAGGCAUGAGCC-3' and reverse, 5'-TCTACGTCG TATCGTCATCTGAC-3'; and U6 forward, 5'-CTCGCTTCG GCAGCACA-3' and reverse, 5'-AACGCTTCACGAATT TGCGT-3'. SYBR ${ }^{\circledR}$ Premix Ex Taq ${ }^{\text {TM }}$ (Tli RNaseH Plus), ROX plus from Takara Biotechnology Co., Ltd. was used according to the manufacturer's protocol, and the total reaction volume was $20 \mu \mathrm{l}$, which contained $10 \mu \mathrm{l}$ SYBR Green premix, $0.3 \mu \mathrm{l}$ correction dye, $1.5 \mu \mathrm{l}$ cDNA, $0.5 \mu \mathrm{l}$ forward primer and $0.5 \mu \mathrm{l}$ reverse primer, and water was added to make a final volume of $20 \mu \mathrm{l}$. The reaction protocol was as follows: $95^{\circ} \mathrm{C}$ for $5 \mathrm{~min}$; 40 cycles at $95^{\circ} \mathrm{C}$ for $15 \mathrm{sec} ; 60^{\circ} \mathrm{C}$ for $15 \mathrm{sec}$; and $72^{\circ} \mathrm{C}$ for 15 sec. qPCR was performed using the Applied Biosystems 7300 Real-Time PCR System (Thermo Fisher Scientific, Inc.). The $2^{-\Delta \Delta \mathrm{Cq}}$ method (20) was used for quantification of the PCR results All assays were performed in triplicate and independently repeated three times.

Statistical analysis. Statistical analysis was performed using SPSS 18.0 software (SPSS, Inc., Chicago, IL, USA) and the values were expressed as the mean \pm standard deviation. Comparisons of continuous data between the two groups were performed using an independent $t$-test, and categorical data were analyzed using the $\chi^{2}$ test or Fisher's exact test. The overall survival (OS) time was calculated as the time between the date of surgery and the date of mortality, or the last follow-up. The disease-free survival (DFS) time was defined as the time between the date of surgery and the date of local or distant recurrence or the date of the last follow-up. Patient survival rates were calculated using the Kaplan-Meier method, and statistically significant differences in survival were identified using the log-rank test. Correlations between the genes were analyzed using Spearman's rank correlation coefficient. $\mathrm{P}<0.05$ was considered to indicate a statistically significant difference.

\section{Results}

MALAT1 expression in CRC tissues and adjacent normal tissues. The expression levels of MALAT1 in 120 CRC tissues and adjacent normal tissues were examined by reverse transcription (RT)-qPCR. The levels of MALAT1 in the CRC tissues were 2.52 times higher compared with the levels in the adjacent normal tissues, which was a significant difference $(\mathrm{P}<0.01$; Fig. 1). These results demonstrated that MALAT1 was evidently upregulated in CRC tumors.

miR-619-5p expression in CRC tissues and adjacent normal tissues. The expression levels of miR-619-5p in $120 \mathrm{CRC}$ tissues and adjacent normal tissues were also determined by RT-qPCR. The levels of miR-619-5p in CRC tissues were 5.79 times lower compared with the levels measured in the adjacent normal tissues, which was a significant difference $(\mathrm{P}<0.01$; Fig. 2). The results demonstrated that miR-619-5p was markedly downregulated in CRC tumors.

Association between MALAT1 expression and the clinicopathological features of CRC patients. Subsequently, the expression of MALAT1 was examined in 120 patients with stage II and III CRC. According to the expression of MALAT1, these cases were divided into the high MALAT1 expression group $(n=60)$ and low expression group $(n=60)$, based on the MALAT1/ GAPDH ratio in CRC tissues. The expression of MALAT1 was significantly increased in male patients compared with female patients $(\mathrm{P}=0.027)$, and MALAT1 expression was significantly associated with the tumor-node-metastasis (TNM) stage $(\mathrm{P}=0.019)$, lymphovascular invasion $(\mathrm{P}=0.047)$ 
Table I. Association between the expression level of MALAT1 and the clinicopathological features of the 120 patients with colorectal cancer.

\begin{tabular}{lccc}
\hline & High MALAT1 & Low MALAT1 & \\
Clinical features & expression, $\mathrm{n}(\%)$ & expression, $\mathrm{n}(\%)$ & P-value
\end{tabular}

Total

$60(100.00)$

$60(100.00)$

Gender

Male

$41(68.33)$

0.027

Female

$19(31.67)$

28 (46.67)

$32(53.33)$

Age

$<65$ years

$37(61.67)$

0.473

$\geq 65$ years

$23(38.33)$

$39(65.00)$

$21(35.00)$

Tumor size, diameter

$\leq 6 \mathrm{~cm}$

$22(36.67)$

0.072

$>6 \mathrm{~cm}$

38 (63.33)

$29(48.33)$

$31(51.67)$

Tumor site

Rectum

$39(65.00)$

$21(35.00)$

$41(68.33)$

Colon

$19(31.67)$

Tumor histology

Adenocarcinoma

43 (71.67)

Mucinous adenocarcinoma

17 (28.33)

$38(63.33)$

$22(36.67)$

Tumor differentiation

Well/moderate

$31(60.00)$

Poor

$29(40.00)$

$36(51.67)$

$24(48.33)$

TNM stage

Stage II

$18(30.00)$

Stage III

$42(70.00)$

$28(46.67)$

$32(53.33)$

Lymph vascular invasion
Absence
$41(68.33)$
Presence
$19(31.67)$

$31(51.67)$

$29(48.33)$

Perineural invasion
Absence
$46(76.67)$
Presence
$14(23.33)$

$33(55.00)$

$27(45.00)$

MALAT1, metastasis associated lung adenocarcinoma transcript 1; TNM, tumor-node-metastasis.

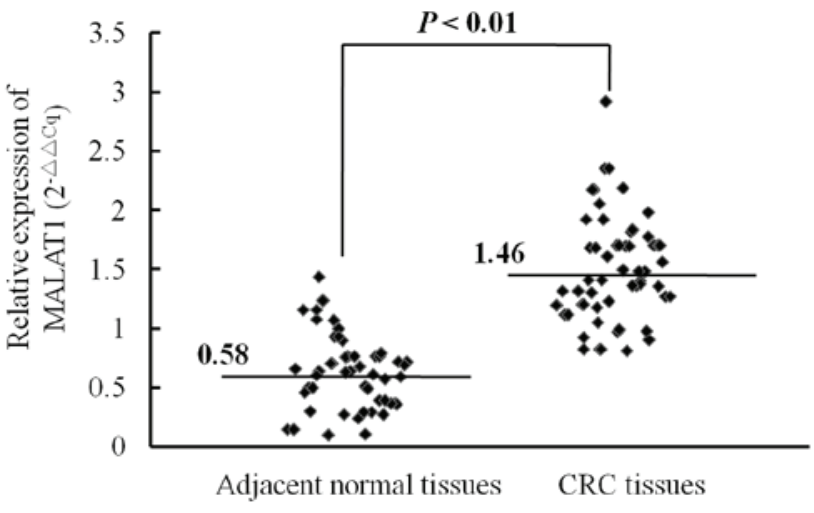

Figure 1. MALAT1 expression in CRC and adjacent normal tissues obtained from 120 patients with CRC. MALAT1 levels were normalized to glyceraldehyde 3-phosphate dehydrogenase. MALAT1 levels in CRC tissues were significantly higher compared with the adjacent normal tissues $(\mathrm{P}<0.01)$. CRC, colorectal cancer; MALAT1, metastasis associated lung adenocarcinoma transcript 1 .

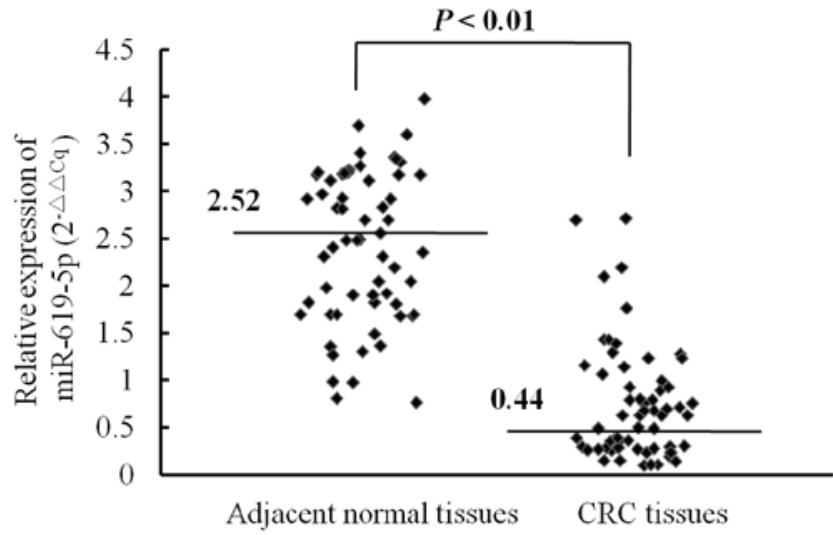

Figure 2. miR-619-5p expression in CRC tissues and adjacent normal tissues obtained from 120 patients with CRC. The miR-619-5p levels were normalized to U6. The miR-619-5p levels in CRC tissues were significantly lower compared with the adjacent normal tissues $(\mathrm{P}<0.01)$. CRC, colorectal cancer; miR, microRNA. 
Table II. Association between miR-619-5p expression and clinicopathological features of the 120 patients with colorectal cancer.

\begin{tabular}{|c|c|c|c|}
\hline Clinical features & High miR-619-5p expression, n (\%) & Low miR-619-5p expression, n (\%) & P-value \\
\hline Total & $60(100.00)$ & $60(100.00)$ & \\
\hline Gender & & & 0.032 \\
\hline Male & $29(48.33)$ & $40(66.67)$ & \\
\hline Female & $31(51.67)$ & $20(33.33)$ & \\
\hline Age & & & 0.373 \\
\hline$<65$ years & $40(66.67)$ & $36(60.00)$ & \\
\hline$\geq 65$ years & $20(33.33)$ & $24(40.00)$ & \\
\hline Tumor size, diameter & & & 0.057 \\
\hline$\leq 6 \mathrm{~cm}$ & $30(50.00)$ & $21(35.00)$ & \\
\hline$>6 \mathrm{~cm}$ & $30(50.00)$ & $39(65.00)$ & \\
\hline Tumor site & & & 0.328 \\
\hline Rectum & $42(70.00)$ & $38(63.33)$ & \\
\hline Colon & $18(30.00)$ & $22(36.67)$ & \\
\hline Tumor histology & & & 0.312 \\
\hline Adenocarcinoma & $39(65.00)$ & $42(70.00)$ & \\
\hline Mucinous adenocarcinoma & $21(35.00)$ & $18(30.00)$ & \\
\hline Tumor differentiation & & & 0.081 \\
\hline Well/moderate & $37(61.67)$ & $30(50.00)$ & \\
\hline Poor & $23(38.33)$ & $30(50.00)$ & \\
\hline TNM stage & & & 0.012 \\
\hline Stage II & $27(45.00)$ & $16(26.67)$ & \\
\hline Stage III & $33(55.00)$ & $44(73.33)$ & \\
\hline Lymph vascular invasion & & & 0.023 \\
\hline Absence & $29(48.33)$ & $39(65.00)$ & \\
\hline Presence & $31(51.67)$ & $21(35.00)$ & \\
\hline Perineural invasion & & & 0.009 \\
\hline Absence & $31(51.67)$ & $45(75.00)$ & \\
\hline Presence & $29(48.33)$ & $15(25.00)$ & \\
\hline
\end{tabular}

miR-619-5p, microRNA; TNM, tumor-node-metastasis.

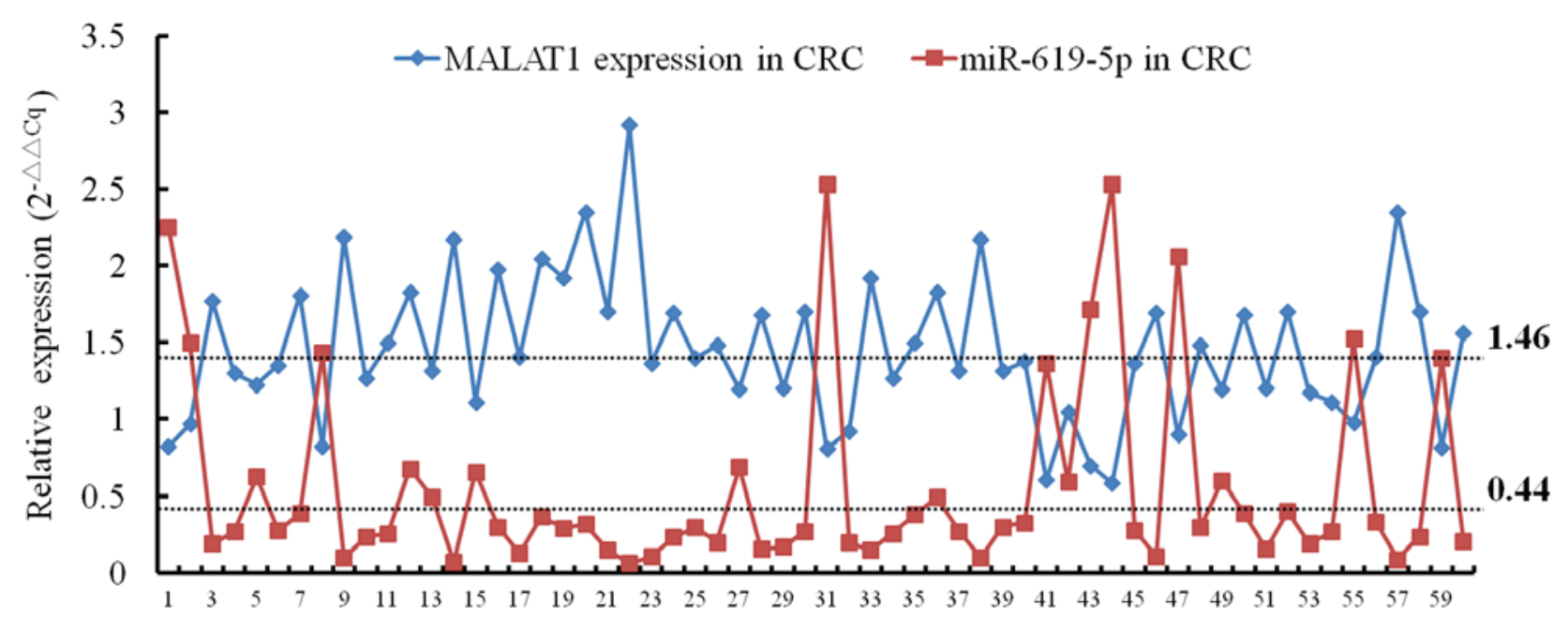

CRC tissue sample number

Figure 3. Comparison between MALAT1 expression and miR-619-5p expression in CRC. The values of 1.46 and 0.44 represent the mean gene expression levels of MALAT1 and miR-619-5p, respectively. MALAT1, metastasis associated lung adenocarcinoma transcript 1; miR-619-5p, microRNA-619-5p; CRC, colorectal cancer. 
Table III. Univariate analysis of DFS and OS for the 120 patients with colorectal cancer.

\begin{tabular}{ccc}
\hline Clinical features & DFS, \\
P-value & OS, \\
P-value
\end{tabular}

MALAT1 expression

$\begin{array}{lll}\text { High } & 0.002 & 0.004\end{array}$

Low

miR-619-5p expression

$\begin{array}{lcc}\text { High } & 0.002 & 0.004 \\ \text { Low } & & \\ \text { Gender } & & \\ \text { Male } & 0.898 & 0.914\end{array}$

\section{Female}

Age

$<65$ years

$\geq 65$ years

Tumor size, diameter

$\leq 6 \mathrm{~cm}$

$>6 \mathrm{~cm}$

Tumor site

Rectum

0.484

Tumor histology

Adenocarcinoma

0.842

Mucinous adenocarcinoma

Tumor differentiation

Well/moderate

Poor

TNM stage

Stage II

Stage III

Lymph vascular invasion

Absence

0.075

Presence

Perineural invasion

Absence

0.001

0.003

Presence

MALAT1, metastasis associated lung adenocarcinoma transcript 1; TNM, tumor-node-metastasis; DFS, disease-free survival; OS, overall survival.

and perineural invasion $(\mathrm{P}=0.012)$, although no association was found between MALAT1 expression and the other clinicopathological features (Table I).

Association between miR-619-5p expression and clinicopathological features of CRC patients. The association between miR-619-5p expression and the clinicopathological features of CRC patients was also investigated. The expression of miR-619-5p was significantly decreased in male patients compared with female patients $(\mathrm{P}=0.032)$, and the expression
Table IV. Spearman's rank correlation analysis of MALAT1 and miR-619-5p expression.

\begin{tabular}{lcr}
\hline & \multicolumn{2}{c}{ Correlation } \\
\cline { 2 - 3 } Parameters & $r$ & P-value \\
\hline $\begin{array}{l}\text { MALAT1 expression } \\
\text { miR-619-5p expression }\end{array}$ & -0.415 & $0.004^{\mathrm{a}}$ \\
\hline
\end{tabular}

${ }^{\mathrm{a}} \mathrm{P}<0.01$ was considered to indicate a statistically significant difference. $r>0.3$ and $r<-0.3$ indicated positive and negative correlations, respectively. MALAT1, metastasis associated lung adenocarcinoma transcript 1; miR-619-5p, microRNA-619-5p.

of miR-619-5p was significantly associated with TNM stage $(\mathrm{P}=0.012)$, lymphovascular invasion $(\mathrm{P}=0.023)$ and perineural invasion $(\mathrm{P}=0.009)$, while no association was identified between miR-619-5p expression and other clinicopathological features (Table II).

Univariate analysis of prognostic factors in patients with stage II and III CRC. The median follow-up period for the 120 patients with $\mathrm{CRC}$ in the present study was 53.6 months, with a range of 10-76.4 months. MALAT1 expression, miR-619-5p expression, TNM stage and perineural invasion were significantly associated with the DFS and OS times (Table III). In particular, patients with a high level of MALAT1 expression or low level of miR-619-5p expression possessed a significantly shorter DFS $(\mathrm{P}=0.002)$ and $\mathrm{OS}(\mathrm{P}=0.004)$ time compared with patients with low MALAT1 expression or a high level of miR-619-5p expression, and patients with perineural invasion demonstrated significantly shorter DFS $(\mathrm{P}=0.001)$ and $\mathrm{OS}(\mathrm{P}=0.003)$ times compared with patients without perineural invasion. Additionally, patients in with TNM stage III CRC also experienced a significantly shorter OS time $(\mathrm{P}=0.037)$ compared with patients with TNM stage II CRC.

Correlation analysis of the MALAT1 and miR-619-5p expression in CRC tissues. Since the upregulated MALAT1 expression and downregulated miR-619-5p expression in CRC tissues were each associated with gender, TNM stage, lymphovascular invasion and perineural invasion, it is essential to investigate the correlation between MALAT1 expression and miR-619-5p expression. As demonstrated in Fig. 3 and Table IV, MALAT1 expression was found to be negatively correlated with miR-619-5p expression $(r=-0.415, \mathrm{P}=0.004)$ in CRC tissues. In general, if MALAT1 expression was high, miR-619-5p expression was low in CRC tissues.

\section{Discussion}

CRC is one of the most common human malignant cancers, which are the synergistic results of various oncogenes and tumor suppressor genes, and remains the third leading cause of cancer-associated death worldwide (1). Although recent advances have improved the diagnosis and therapy of patients with $\mathrm{CRC}$, there are few reliable markers available to 
accurately predict metastasis in CRC patients, particularly in patients with early-stage CRC.

Previously, lncRNAs have been increasingly reported to be involved in human disease $(21,22)$. MALAT1 is a highly conserved and newly identified lncRNA, which was first found to be overexpressed in metastatic non-small cell lung cancer (NSCLC) (9). Subsequently, MALAT1 was found to be associated with several other cancers, including cervical, hepatic, breast and renal cancer $(10,11,23-26)$, and was considered to regulate tumor growth, invasion and migration in different types of cancers (27-30).

In addition, short ncRNAs, such as miRNAs, have been the focus of studies, and numerous miRNAs have shown extremely important effects in the development of cancers. miR-619-5p is a miRNA that binds with high affinity to the mRNAs of 1,215 genes, and miR-619-5p has binding sites in the coding sequences and UTRs of mRNAs (19). Bioinformatics analysis indicated that miR-619-5p has several binding sites on the 3'-UTR of MALAT1. However, the association between MALAT1 and miR-619-5p in CRC is not well studied.

The present study examined the expression of MALAT1 and miR-619-5p in CRC tissues and adjacent normal tissues. The analysis indicated that there were significant differences between MALAT1 expression in CRC tissues and adjacent normal tissues, which were consistent with previously reported results $(13,14,31)$, and to the best of our knowledge, miR-619-5p expression was reported to be significantly different between CRC tissues and adjacent normal tissues for the first time in the present study.

Investigation of the molecular diagnosis of CRC may broaden the scope of medical research. The present results have demonstrated that patients with high MALAT1 expression and low miR-619-5p expression had a significantly increased risk of metastasis, such as lymphovascular and perineural invasion, subsequent to radical surgery. MALAT1 was first found to be associated with tumor metastasis in patients with NSCLC, and knockdown of MALAT1 in A549 lung cancer cells inhibits cell migration without affecting cell proliferation (30). Additional mechanism analysis revealed that MALAT1 is critical for the Wnt/ $\beta$-catenin signaling pathway and releasing the oncogene polypyrimidine tract binding protein-2 (PTBP-2) from the splicing factor proline/glutamine-rich/PTBP-2 complex $(13,14)$. Overall, these mechanisms may explain the strong tendency for metastasis subsequent to surgery in patients with high expression of MALAT1. In addition, miR-619-5p has been found to be extremely important in the metastasis of CRC, whose function and mechanism required additional investigation. Since miR-619-5p has several binding sites on the 3'-UTR of MALAT1, assessment of the function and mechanism of miR-619-5p may be extremely beneficial in future studies.

Univariate Cox regression analysis indicated that MALAT1 expression, miR-619-5p expression and perineural invasion were independent predictors of the DFS and OS times, and tumor TNM stage and lymphovascular invasion were also found to be independent predictors of the OS time.

Since upregulated MALAT1 expression and downregulated miR-619-5p expression in CRC tissues were each associated with the TNM stage, metastasis, DFS time and OS time, the correlation between MALAT1 expression and miR-619-5p expression was investigated. Spearman's rank correlation analysis revealed a negative correlation between the differential expression of MALAT1 and miR-619-5p ( $r=-0.346$; $\mathrm{P}=0.030$ ), suggesting that combined detection of miR-619-5p and MALAT1 may improve the accuracy of the diagnosis of $\mathrm{CRC}$, and the expression of miR-619-5p and MALAT1 may act as a good prognostic indicator in CRC patients.

In conclusion, upregulated MALAT1 expression and downregulated miR-619-5p expression may be involved in the progression of CRC and may therefore be considered a diagnostic marker and prognostic factor for patients with stage II or III CRC. The identification of this novel biomarker may aid the understanding of the possible molecular mechanisms underlying the recurrence and metastasis of CRC, and provide additional therapeutic targets for CRC patients.

\section{References}

1. Jemal A, Bray F, Center MM, Ferlay J, Ward E and Forman D: Global cancer statistics. CA Cancer J Clin 61: 69-90, 2011.

2. Matouk IJ, DeGroot N, Mezan S, Ayesh S, Abu-lail R, Hochberg A and Galun E: The H19 non-coding RNA is essential for human tumor growth. PLoS One 2: e845, 2007.

3. Huarte M, Guttman M, Feldser D, Garber M, Koziol MJ, Kenzelmann-Broz D, Khalil AM, Zuk O, Amit I, Rabani M, et al: A large intergenic noncoding RNA induced by p53 mediates global gene repression in the p53 response. Cell 142: 409-419, 2010.

4. Pasmant E, Sabbagh A, Masliah-Planchon J, Ortonne N, Laurendeau I, Melin L, Ferkal S, Hernandez L, Leroy K, Valeyrie-Allanore L, et al: Role of noncoding RNA ANRIL in genesis of plexiform neurofibromas in neurofibromatosis type 1 . J Natl Cancer Inst 103: 1713-1722, 2011.

5. Zhou Y, Zhang X and Klibanski A: MEG3 noncoding RNA: A tumor suppressor. J Mol Endocrinol 48: R45-R53, 2012.

6. Du Y, Kong G, You X, Zhang S, Zhang T, Gao Y, Ye L and Zhang X: Elevation of highly up-regulated in liver cancer (HULC) by hepatitis B virus $\mathrm{X}$ protein promotes hepatoma cell proliferation via down-regulating p18. J Biol Chem 287: 26302-26311, 2012.

7. Ling H, Spizzo R, Atlasi Y, Nicoloso M, Shimizu M, Redis RS, Nishida N, Gafà R, Song J, Guo Z, et al: CCAT2, a novel noncoding RNA mapping to $8 \mathrm{q} 24$, underlies metastatic progression and chromosomal instability in colon cancer. Genome Res 23: 1446-1461, 2013.

8. Li CH and Chen Y: Targeting long non-coding RNAs in cancers: Progress and prospects. Int J Biochem Cell Biol 45: 1895-1910, 2013.

9. Ji P, Diederichs S, Wang W, Böing S, Metzger R, Schneider PM, Tidow N, Brandt B, Buerger H, Bulk E, et al: MALAT-1, a novel noncoding RNA and thymosin beta4 predict metastasis and survival in early-stage non-small cell lung cancer. Oncogene 22: 8031-8041, 2003.

10. Yamada K, Kano J, Tsunoda H, Yoshikawa H, Okubo C, Ishiyama T and Noguchi M: Phenotypic characterization of endometrial stromal sarcoma of the uterus. Cancer Sci 97: 106-112, 2006.

11. Lin R, Maeda S, Liu C, Karin M and Edgington TS: A large noncoding RNA is a marker for murine hepatocellular carcinomas and a spectrum of human carcinomas. Oncogene 26: 851-858, 2007.

12. Tseng JJ, Hsieh YT, Hsu SL and Chou MM: Metastasis associated lung adenocarcinoma transcript 1 is up-regulated in placenta previa increta/percreta and strongly associated with trophoblast-like cell invasion in vitro. Mol Hum Report 15: 725-731, 2009.

13. Ji Q, Liu X, Fu X, Zhang L, Sui H, Zhou L, Sun J, Cai J, Qin J, Ren $\mathrm{J}$ and Li Q: Resveratrol Inhibits invasion and metastasis of colorectal cancer cells via MALAT1 mediated Wnt $/ \beta$-catenin signal pathway. PLoS One 8: e78700, 2013.

14. Ji Q, Zhang L, Liu X, Zhou L, Wang W, Han Z, Sui H, Tang Y, Wang Y, Liu N, et al: Long non-coding RNA MALAT-1 promotes tumor growth and metastasis in colorectal cancer through binding to SFPQ and releasing oncogene PTBP-2 from SFPQ/PTBP-2 complex. Br J Cancer 111: 736-748, 2014. 
15. Skaftnesmo KO, Prestegarden L, Micklem DR and Lorens JB: MicroRNAs in tumorigenesis. Curr Pharm Biotechnol 8: 320-325, 2007

16. Hernando E: microRNAs and cancer: Role in tumorigenesis, patient classification and therapy. Clin Transl Oncol 9: 155-160, 2007.

17. Faragalla H, Youssef YM, Scorilas A, Khalil B, White NM, Mejia-Guerrero S, Khella H, Jewett MA, Evans A Lichner Z, et al: The clinical utility of miR-21 as a diagnostic and prognostic marker for renal cell carcinoma. J Mol Diagn 14 385-392, 2012

18. Koberle V, Kronenberger B, Pleli T, Trojan J, Imelmann E, Peveling-Oberhag J, Welker MW, Elhendawy M, Zeuzem S, Piiper A and Waidmann O: Serum microRNA-1 and microRNA-122 are prognostic markers in patients with hepatocellular carcinoma. Eur J Cancer 49: 3442-3449, 2013.

19. Ivashchenko A, Berillo O, Pyrkova A, Niyazova R and Atambayeva S: The properties of binding sites of miR-619-5p, miR-5095, miR-5096 and miR-5585-3p in the mRNAs of human genes. Biomed Res Int 2014: 720715, 2014.

20. Livak KJ and Schmittgen TD: Analysis of relative gene expression data using real-time quantitative PCR and the 2(-Delta Delta C(T)) method. Methods 25: 402-408, 2001

21. Wapinski $\mathrm{O}$ and Chang HY: Long noncoding RNAs and human disease. Trends Cell Biol 21: 354-361, 2011.

22. Esteller M: Non-coding RNAs in human disease. Nature Rev Genet 12: 861-874, 2011

23. Luo JH, Ren B, Keryanov S, Tseng GC, Rao UN, Monga SP, Strom S, Demetris AJ, Nalesnik M, Yu YP, et al: Transcriptomic and genomic analysis of human hepatocellular carcinomas and hepatoblastomas. Hepatology 44: 1012-1024, 2006
24. Guffanti A, Iacono M, Pelucchi P, Kim N, Soldà G, Croft LJ, Taft RJ, Rizzi E, Askarian-Amiri M, Bonnal RJ, et al: A transcriptional sketch of a primary human breast cancer by 454 deep sequencing. BMC Genomics 10: 163, 2009.

25. Praz V, Jagannathan V and Bucher P: CleanEx: A database of heterogeneous gene expression data based on a consistent gene nomenclature. Nucleic Acids Res 32 (Database issue): D542-D547, 2004.

26. Davis IJ, Hsi BL, Arroyo JD, Vargas SO, Yeh YA, Motyckova G, Valencia P, Perez-Atayde AR, Argani P, Ladanyi M, et al: Cloning of an Alpha-TFEB fusion in renal tumors harboring the $\mathrm{t}(6 ; 11)$ (p21;q13) chromosome translocation. Proc Natl Acad Sci USA 100: 6051-6056, 2003

27. Lai MC, Yang Z, Zhou L, Zhu QQ, Xie HY, Zhang F, Wu LM, Chen LM and Zheng SS: Long non-coding RNA MALAT-1 overexpression predicts tumor recurrence of hepatocellular carcinoma after liver transplantation. Med Oncol 29: 1810-1816, 2012.

28. Schmidt LH, Spieker T, Koschmieder S, Schäffers S, Humberg J, Jungen D, Bulk E, Hascher A, Wittmer D, Marra A, Hillejan L, et al: The long noncoding MALAT-1 RNA indicates a poor prognosis in non-small cell lung cancer and induces migration and tumor growth. J Thorac Oncol 6: 1984-1892, 2011.

29. Gutschner T, Hämmerle $M$ and Diederichs S: MALAT1-a paradigm for long noncoding RNA function in cancer. J Mol Med (Berl) 91: 791-801, 2013.

30. Gutschner T, Hämmerle M, Eissmann M, Hsu J, Kim Y, Hung G, Revenko A, Arun G, Stentrup M, Gross M, et al: The noncoding RNA MALAT1 is a critical regulator of the metastasis phenotype of lung cancer cells. Cancer Res 73: 1180-1189, 2013.

31. Zheng HT, Shi DB, Wang YW, Li XX, Xu Y, Tripathi P, Gu WL, Cai GX and Cai SJ: High expression of lncRNA MALAT1 suggests a biomarker of poor prognosis in colorectal cancer. Int J Clin Exp Pathol 7: 3174-3181, 2014. 